EPV118/\#381 THE INCREASED INCIDENCE OF UTERINE CANCER WITH HIGH RISK HISTOLOGIES - A POPULATION STUDY FROM THE TAIWAN CANCER REGISTRY

${ }^{1} \mathrm{C}-\mathrm{I}$ Liao, ${ }^{2} \mathrm{M}$ Richardson, ${ }^{3} \mathrm{C}$ Tian, ${ }^{3} \mathrm{~K}$ Darcy, ${ }^{4} \mathrm{D}$ Kapp, ${ }^{5} \mathrm{~J}$ Chan. ${ }^{1}$ Kaohsiung Veterans General Hospital, Obstetrics and Gynecology, Kaohsiung City, Taiwan; ${ }^{2}$ University of California, Los Angeles, Obstetrics and Gynecology, Los Angeles, USA; ${ }^{3}$ Gynecologic Cancer Center of Excellence, Obstetrics and Gynecology, Washington, USA; ${ }^{4}$ Stanford University School of Medicine, Department of Radiation Oncology, Stanford, USA; ${ }^{5}$ California Pacific Medical Center, Obstetrics and Gynecology, San Francisco, USA

\subsection{6/ijgc-2021-IGCS.188}

Objectives To evaluate trends in uterine cancer diagnosis and incidence by histology in Taiwan between 2001-2017.

Methods Data were obtained from the Taiwan Cancer Registry of Taiwan Health and Welfare Data Center for women diagnosed with a malignancy of the uterine corpus from 2001 to 2017. Joinpoint regression analysis was used to evaluate and project trends over time.

Results There were 26,827 women in Taiwan Cancer Registry diagnosed with uterine cancer between 2001-2017, including $25.2 \%$ with grade (G)1-endometrioid endometrial carcinoma (EEC), 36.5\% with G2-EEC, 25.2\% G3-EEC, 3.5\% with uterine serous carcinoma (USC), 3.4\% with uterine carcinosarcoma (UCS) and 2.1\% with uterine clear cell carcinoma (UCCC). The proportion of women with a high-risk histology defined as G2-EEC, G3-EEC, USC, UCS, or UCCC increased from $51 \%$ to $63 \%$ when diagnosed at $50-59$ or $60-69$ years of age, respectively. The average incidence per 100,000 by histology was 9.2 with EEC, 0.64 with USC, 0.51 with UCS, and 0.25 with UCCC. The annual percent change (APC) in incidence between 2001 and 2017 increased by 10.6\% for a USC diagnosis, $5.8 \%$ for a UCS diagnosis, and $4.6 \%$ for a UCCC diagnosis. Predictive modeling projects that the incidence of USC in women between 60-64 years old will surpass G1-EEC incidence in the same age group by 2022 .

Conclusions High-risk uterine cancers constitute a substantial portion of the uterine cancers in the Taiwan Cancer Registry, particularly for women in their 60 s or older. This exponential rise has important health and welfare implications in Taiwan and the International community.

\section{EPV119/\#397 VOLUME OF NODAL DISEASE AND ONCOLOGIC OUTCOMES IN ENDOMETRIAL CANCER PATIENTS WITH POSITIVE SENTINEL LYMPH NODES: AN ITALIAN MULTI-INSTITUTIONAL STUDY}

\begin{abstract}
${ }^{1} \mathrm{~F}$ Martinelli*, ${ }^{2} \mathrm{~A}$ Buda, ${ }^{3} \mathrm{~F}$ Fanfani, ${ }^{4} \mathrm{~F}$ Legge, ${ }^{5} \mathrm{M}$ Roccio, ${ }^{6} \mathrm{~F}$ Falcone, ${ }^{7} \mathrm{AM}$ Perrone, ${ }^{8} \mathrm{~J}$ Casarin, ${ }^{9} \mathrm{~A}$ Perutelli, ${ }^{10} \mathrm{~N}$ Biglia, ${ }^{11} \mathrm{P}$ Scollo, ${ }^{12} \mathrm{~F}$ Romano, ${ }^{1} \mathrm{~A}$ Ditto, ${ }^{2} \mathrm{D}$ Ferrari, ${ }^{3} \mathrm{G}$ Monterossi, ${ }^{4} \mathrm{~F}$ Murgia, ${ }^{2} \mathrm{C}$ Paniga, ${ }^{3} \mathrm{G}$ Scambia, ${ }^{1} \mathrm{~F}$ Raspagliesi. ${ }^{1}$ Fondazione IRCCS Istituto Nazionale Tumori of Milan, Gynecologic Oncology, Milan, Italy; ${ }^{2}$ University of Milan Bicocca, Uoc of Gynecology, Milan, Italy; ${ }^{3}$ Università Cattolica del Sacro Cuore, Department of Woman and Child Health and Public Health, Woman Health Area, Fondazione Policlinico Universitario A. Gemelli Irccs, Roma, Italy; ${ }^{4}$ 'F. Miulli' General Regional Hospital, Gynecologic Oncology Unit, Acquaviva Delle Fonti, Italy; ${ }^{5}$ Fondazione IRCCS Policlinico San Matteo and University of Pavia, Department of Obstetrics and Gynecology, Pavia, Italy; ${ }^{6}$ Endoscopica Malzoni, Center for Advanced Endoscopic Gynecological Surgery, Gynecological Surgery, Avellino, Italy; ' University of Bologna, Gynecologic Oncology, Bologna, Italy; ${ }^{8}$ University of Insubria, Gynecologic Oncology, Varese, Italy; ${ }^{9}$ Azienda Ospedaliero Universitaria Pisana, Uo Ginecologia 2, Pisa, Italy; ${ }^{10} A$. O. Ordine Mauriziano Umberto I, Ginecologia E Ostetricia, Torino, Italy; ${ }^{11}$ Cannizzaro Hospital of Catania, Unit of Obstetrics and Gynaecology, Catania, Italy; ${ }^{12}$ Institute for Maternal and Child Health 'IRCCS Burlo Garofolo', Clinica Ostetrica E Ginecologica, Trieste, Italy
\end{abstract}

10.1136/ijgc-2021-IGCS.189
Objectives To asses predictive factors for sentinel-lymph-nodes (SLNs) involvement and recurrence-free-survival (RFS) in patients with endometrial cancer

Methods A multicenter retrospective evaluation of endometrial-cancer patients with positive (macro-micro metastases or ITCs) SLNs, treated between 2003 and 2020, was performed. Predictive factors for nodal involvement (endometrioid vs nonendometrioid histology, grading, lymphovascular-space-invasion (LVSI), myometrial-invasion (MI), cervical-stromal-invasion, ESGO/ESTRO/ESP risk group), adjuvant therapy and oncological outcomes were evaluated

Results 142 patients were identified among 12 participating centers. In $64.8 \%$ of cases a low-volume disease $(\leq 2 \mathrm{~mm})$ was found in SLNs: 33 (23.2\%) ITCs and 59 (41.6\%) micrometastases. Predictors of macrometastatic SLNs were: high grade [p:0.002], LVSI [p:0.007] and MI $>50 \%$ [p:0.008]. 17 $(18.5 \%)$ patients with low-volume disease $(8$ micrometastases and 9 ITCs) did not receive any adjuvant therapy. At a mean follow-up of 34.6 months (range 1-215) months, 21 (14.8\%) relapses were recorded, only one among patients not receiving any adjuvant.The RFS at 2-years for the micrometastatic patients was 91\%, similar to ITCs patients $(79.1 \%)$, regardless of adjuvant treatment, but statistically better than patients with macrometastases (72.3\%) [p: 0.026].The only factors affecting RFS were deep MI [p:0.03] and cervical stromal invasion [p:0.046].

Conclusions More than half of patients with positive SLNs had low-volume disease. Grading, MI and LVSI predicted volume of nodal metastases. MI and cervical invasion affected RFS; while adjuvant treatment did not seem significantly associated with RFS in patients with low-volume disease. Longer follow-up time and a larger sample size are needed to understand the role of adjuvant therapy in low-volume metastatic SLNs.

\section{EPV120/\#399 ENDOMETRIAL CANCER: MOLECULAR ANALYSIS AND CLINICOPATHOLOGICAL CORRELATION: A PILOT STUDY}

'A Dahiya*, 'A Thomas, ${ }^{2} \mathrm{R}$ Pai, ${ }^{3} \mathrm{~S}$ Daniel, ${ }^{1} \mathrm{~A}$ Sebastian, ${ }^{1} \mathrm{D}$ Susan Thomas, ${ }^{1} \mathrm{~V}$ Thomas, ${ }^{1} \mathrm{R}$ Chandy, ${ }^{4} \mathrm{~T}$ Samuel Ram, ${ }^{4} \mathrm{~A}$ S, ${ }^{5} \mathrm{G}$ Rebekah, ${ }^{1} \mathrm{~A}$ Peedicayil. ${ }^{1}$ Christian Medical College, Vellore, Gynecologic Oncology, Vellore, India; ${ }^{2}$ Christian Medical College, Vellore, Molecular Pathology, Vellore, India; ${ }^{3}$ Christian Medical College, Vellore, Pathology, Vellore, India; ${ }^{4}$ Christian Medical College, Vellore, Radiation Oncology, Vellore, India; ${ }^{5}$ Christian Medical College, Vellore, Biostatistics, Vellore, India

\subsection{6/ijgC-2021-IGCS. 190}

Objectives Aim/Introduction: Limited reproducibility and imprecise risk estimation of traditional classification have paved the way for molecular research in endometrial cancer. The study aims to determine the prevalence of Polymerase Epsilon gene (POLE) mutation, P53 mutations, and microsatellite instability (MSI) in endometrial cancer, followed by clinicopathological correlation.

Methods Materials and Methods: A retrospective cohort involving 50 consecutive patients of primary endometrial carcinoma was identified from 01.01.2016 to 01.02.2018 using the computerized database. Molecular classification of endometrial cancer was done with the following components. POLE ultramutated: using exon 9-14 mutational analysis, Microsatellite instability (MSI) using Mismatch repair protein IHC (MLH1, MSH2, MSH6), and Copy number high/low: using p53 IHC as a surrogate marker. 


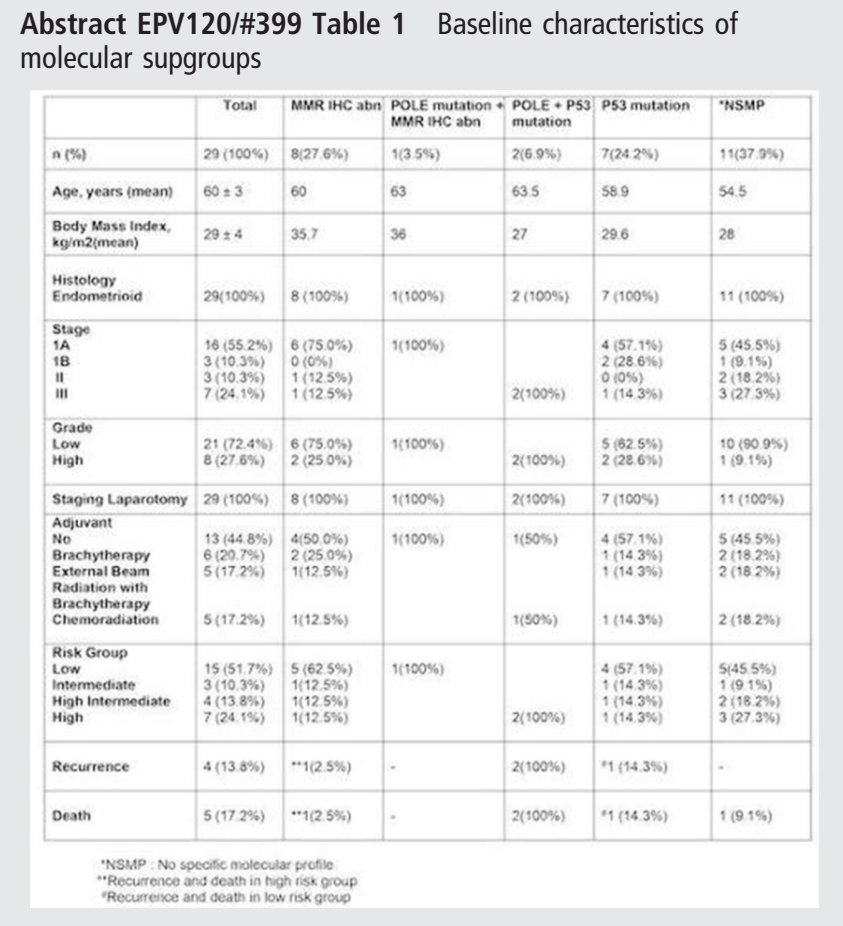

Results Results/Conclusions An interim analysis of 29 patients was done. Eight $(27.6 \%)$ patients had MLH1 mutation, 1 (3.5\%) patient had POLE and MLH1 mutation, while 2 (6.9\%) had both POLE and P53 mutation. Seven (24.2\%) patients were found to have null mutations of P53, while the remaining 11 (37.9\%) had no specific molecular profile (NSMP). ESMO-ESGO risk group correlation, recurrences, and deaths are shown in table 1 .

Conclusions Implications: Recurrence in low risk groups, behaviour of multiple classifiers, NSMP group and POLE mutated higher risk/stage cancers are areas still underresearched. A larger study exploring the integrated approach will help answer these questions and open novel avenue of research aimed at immunotherapy in endometrial cancer especially in recurrent settings.

\section{EPV121/\#419 BASELINE CLINICAL OUTCOMES OF LYNCH SYNDROME PATIENTS UNDERGOING ANNUAL SURVEILLANCE VERSUS RISK-REDUCING SURGERY IN A PROSPECTIVE COHORT STUDY}

${ }^{1} \mathrm{~S}$ Piedimonte*, ${ }^{2} \mathrm{~A}$ Zia, ${ }^{3} \mathrm{M}$ Maganti, ${ }^{2} \mathrm{~S}$ Ferguson. 'University of Toronto, Gynecologic Oncolgoy, Toronto, Canada; ${ }^{2}$ Princess Margaret Cancer Centre/University of Health Network/Sinai Health Systems, Gynecologic Oncology, Toronto, Canada; ${ }^{3}$ Princess Margaret Cancer Centre, Biostatistics, Toronto, Canada

\subsection{6/ijgc-2021-IGCS.191}

Objectives To describe baseline characteristics of Lynch syndrome(LS) patients enrolled in a prospective study of annual surveillance versus risk-reducing surgery(RRS) and determine prevalent cases of endometrial intraepithelial neoplasia(EIN), endometrial(EC) and ovarian(OC) cancers

Methods A prospective cohort study was implemented in February 2015 for LS patients diagnosed based on a pathogenic variant in mismatch repair genes but unaffected by gynecologic cancer. Baseline investigations included CA-125, ultrasound and endometrial biopsy(EMB); further investigations were performed as warranted. Patients were recommended RRS by age 40 or following child-bearing. All others had annual surveillance and analyzed per treatment received.

Results Among 82 patients, 41 underwent RRS and 41 annual surveillance. The most frequent mutation was MSH6(34.1\%). $25.9 \%$ had a personal history of LS-associated cancer and 97.5\% had a family history, most commonly being colorectal (74.4\%). Patients in the RRS group had a higher median age at LS diagnosis(47 vs 32 years, $p<0.001)$ and entry into LS screening program(47 vs 33 years, $\mathrm{p}<0.001)$. At baseline, median CA-125 was 10 in both groups $(\mathrm{p}=0.65)$. The baseline EMB rate was $85 \%(n=70)$ with an abnormality rate of $4.88 \%$ (two EIN in surveillance group and one EC in RRS group). Seventy(91\%) individuals underwent baseline ultrasound and no OCs were detected. In patients undergoing RRS, the median time from initial visit to surgery was 6.1 months(range 1.1-20.7); 3 additional EINs were diagnosed on final pathology.

Conclusions In LS patients followed in a surveillance program, the prevalent rate of EIN/EC is $5-10 \%$, mostly in the RRS group. RRS within the recommended time prevents diagnosis of significant pathology.

\section{EPV122/\#421 ISOLATED LYMPHATIC RECURRENCE IN ENDOMETRIAL CANCER: A RETROSPECTIVE STUDY}

${ }^{1} \mathrm{~S}$ Garzon, ${ }^{1} \mathrm{~S}$ Kumar, ${ }^{2} \mathrm{~A}$ Weaver, ${ }^{2} \mathrm{M}$ Mcgree, ${ }^{3} \mathrm{~S}$ Uccella, ${ }^{4} \mathrm{G}$ Keeney, ${ }^{1} \mathrm{~W}$ Cliby, ${ }^{1} \mathrm{~A}$ Mariani. ${ }^{1}$ Mayo Clinic, Department of Obstetrics and Gynecology, Rochester, USA; ${ }^{2}$ Division of Biomedical Statistics and Informatics, Department of Health Sciences Research, Rochester, USA; ${ }^{3}$ AOUI Verona, University of Verona, Department of Obstetrics and Gynecology, Verona, Italy; ${ }^{4}$ Mayo Clinic, Department of Laboratory Medicine and Pathology, Rochester, USA

\subsection{6/ijgc-2021-IGCS.192}

Objectives We investigated factors associated with cause-specific survival (CSS) after isolated lymphatic recurrence (ILR) in endometrial cancer (EC).

Methods We identified patients who developed ILR among 4,216 EC patients surgically treated at the Mayo Clinic between 1984 and 2017. ILR was defined as the first and unique evidence of recurrence in lymph node-bearing areas (with or without $( \pm)$ vaginal recurrence). Univariate and multivariable Cox regression analysis was used to evaluate factors associated with CSS after ILR.

Results We observed 70 cases of ILR: 12 pelvic, 15 paraaortic, 14 pelvic and paraaortic, and 29 distant ( \pm pelvic and/or paraaortic). Most women (90.0\%) underwent pelvic and/or paraaortic lymphadenectomy during primary surgery, and $68.3 \%$ had positive nodes. Among 70 patients, 50 died of disease with median survival after ILR of 1.4 years. Patients who did not die of EC had a median follow-up after ILR of 6.6 (IQR 4.8-10.0) years. By univariate analysis, histologic grade, lymphovascular space invasion, ILR site, concomitant vaginal recurrence, and ILR treatment were significantly associated with CSS after ILR. CSS after ILR was not associated with primary lymphadenectomy, stage, or adjuvant therapy. Results of the multivariable analysis are reported in the Table.

Conclusions Histologic grade 2 or 3 of the primary tumor and concomitant recurrence in the pelvic and paraaortic lymph node basins or at the vaginal cuff were independent 